\title{
Perspectiva de género y
}

privación de libertad: algunas

re exiones sobre una (reciente

y necesaria) reforma al Código

Penal Costarricense

Gender Perspective and Deprivation of Liberty: Some Re ections on a (Recent and Necessary) Reform of the Costa Rican Penal Code

José Daniel Mora Bolaños ${ }^{1}$

Asamblea Legislativa

\section{Resumen}

Costa Rica

Según diversos estudios, la mayoría de mujeres que delinquen tienen características particulares de vulnerabilidad -como pobreza, violencia o dependencia a cargo- que pueden explicar la infracción al ordenamiento jurídico. Estos factores han sido históricamente ignorados por los sistemas de justicia, lo cual supone un elevado riesgo de mayor exclusión y marginalidad. A principios del 2019, Costa Rica aprobó una reforma legal para incorporar la perspectiva de género en la sanción a mujeres en conflicto con la ley penal. Esta reforma permitirá que los jueces puedan rebajar el monto de la pena cuando haya mediado una condición de vulnerabilidad. El presente artículo, a través de una revisión bibliográfica y legislativa, busca poner en valor el cambio normativo y explicar sus alcances. También se presenta una revisión sobre el perfil femenino de la criminalidad y se plantean algunos desafíos del sistema penitenciario en la atención de las mujeres, especialmente, las prisionalizadas.

Palabras clave: mujeres; sistema penitenciario; vulnerabilidad; pena; perspectiva de género.

1 Estudió Derecho y Ciencias Políticas en la Universidad de Costa Rica. Criminólogo de la Universidad

Nacional Estatal a Distancia (UNED). Correo electrónico:jose.mora@asamblea.go.cr 


\begin{abstract}
According to several studies, the majority of women offenders live in particular conditions of vulnerability - such as poverty, violence, or dependency - that can explain the violation of the legal system. These factors have historically been ignored by justice systems, which poses a high risk of further exclusion and marginalization. In early 2019, Costa Rica approved a legal reform to incorporate a gender perspective in the punishment of women in conflict with the criminal law. This reform will allow judges to reduce the amount of the sentence when there has been a condition of vulnerability. Through a bibliographic and legislative review, this article seeks to value the normative change and explain its scope. It also presents an analysis of the feminine profile of criminality and poses some challenges to the penitentiary system in the care of women, especially those imprisoned.
\end{abstract}

Keywords: women; prison system; vulnerability; punishment; gender perspective.

\title{
A. Introducción
}

María tiene 3 hijos, hace 4 años quedó viuda, así, sola, sin ningún respaldo económico, viviendo en Puntarenas, basculaba entre la desesperación de mantener a los menores y la obligación de respetar las normas jurídicas. Un "conocido" le propuso vender drogas, llevaba meses desempleada, y esa, pensó, sería una forma sencilla, y en apariencia solo temporal, de resolver los ingentes problemas que la tenían al borde de la desesperación. La temporalidad acabó cuando la policía judicial la detuvo y se le impuso una pena de 7 años de prisión (Feoli, 2019).

Nadie duda de que en el marco de un Estado de Derecho las conductas que el legislador ha considerado delictivas deben ser investigadas y sancionadas cuando así corresponda. Al tiempo, existen casos matizados y cruzados, por una realidad de exclusión que cuando se limitan a la simple imposición de un castigo, tan severo como la privación de la libertad, pueden traer, junto al respeto formal por el ordenamiento jurídico, más injusticia y marginalidad.

La población penal ubicada en centros cerrados, según datos del sistema penitenciario nacional, es de 15454 personas, de las cuales 647 son mujeres. Esto representa un 4,1 \%. (Dirección General de Adaptación Social, 2018). Sin embargo, supone uno de los grupos históricamente vulnerables que requieren de una atención especializada por parte del Estado. Por esta razón, en línea con lo que han demandado algunos instrumentos internacionales de derechos humanos, Costa Rica aprobó una reforma al Código Penal, en noviembre de 2018, que permite 
que las condiciones de vulnerabilidad que influyen en la comisión del delito cometido por mujeres puedan ser valoradas por los jueces.

En el presente artículo, se busca como objetivo principal explicar el impacto y la importancia que debe tener la reforma a los artículos 71 y 72 del Código Penal. Para ello, se analizan las condiciones particulares que explican el encierro femenino, pero también las razones por las que se requiere de un abordaje diferenciado desde el Estado. El paper se ha dividido en tres apartados; el primero se denomina: "Mujeres y encierro" y trata aspectos relacionados con la prisionalización de las mujeres; en el segundo, "La reforma esperada o la expresión de un Nuevo Humanismo“, donde se presentan los antecedentes que permitieron alcanzar la modificación de los artículos 71 y 72 de la ley penal costarricense. Finalmente, el tercer acápite propone algunos desafíos pendientes y líneas de acción que convendría plantearse en el marco de la reforma y de la necesidad de repensar la atención penitenciaria de las mujeres en conflicto con la ley penal.

\section{B. Mujeres y encierro}

Más allá de textos legales, la igualdad sustantiva entre mujeres y hombres es un objetivo que se debe definir en las líneas de acción de las políticas públicas. Sin embargo, las relaciones humanas en sociedades que han sido estructuradas a partir del ejercicio del poder de dominio, se desenvuelven en un contexto que genera desigualdad. Esa desigualdad coloca a las mujeres, por mandatos estructurales sobre cómo deben ser y lo que deben hacer, en desventaja en todos los ámbitos de la vida (Facio y Fries, 2005). Es importante entonces conocer las aristas particulares que expresa la vulnerabilidad que enfrentan las mujeres por su condición de género. En ese sentido:

(...) se entiende en situación de vulnerabilidad a las mujeres que entran en contacto con el sistema penal, quienes, en razón de los condicionamientos culturales y sociales, tienen bajo su responsabilidad directa a personas con enfermedades, menores de edad, adultas mayores, entre otras, de manera que su detención genera un impacto personal hacia ellas, sus dependientes y por ende, hacia la sociedad. (Poder Judicial, 2018)

Las disciplinas que abordan el fenómeno criminal no pueden obviar la realidad sociocultural de las mujeres. Así, desde el derecho penal, la criminología y las políticas penitenciarias, deben tomarse en consideración las enormes diferencias que existen entre las mujeres y hombres que delinquen (Davis, 2016). 
¿Cómo se refleja la vulnerabilidad? La información disponible advierte que una inmensa mayoría de las mujeres que enfrentan el encarcelamiento son las responsables directas de sus familiares, especialmente de sus hijos e hijas. En el 2015, en el "Diagnóstico de necesidades de mujeres privadas de libertad en el Centro de Atención Institucional (CAI) Buen Pastor" (hoy llamado Vilma Curling Rivera), se entrevistó a una muestra de 100 mujeres. Los resultados son contundentes: el $85 \%$ de las mujeres entrevistadas reportó tener hijos e hijas, con un promedio de tres que en su mayoría dependían de ellas antes del ingreso a la cárcel.

Otro trabajo, realizado en 2016 por la Fundación Arias para la Paz y el Progreso Humano, encuestó a 150 mujeres. Según informaron, un $60 \%$ de los familiares que dependen de ellas son sus hijos menores, seguido por un $18 \%$ que corresponde a padres y, atrás, un 11 \% que tienen a cargo a sus nietos (Fundación Arias para la Paz y el Progreso Humano, 2016).

Muchas mujeres, sobre todo las que tienen la jefatura del hogar, dadas las dificultades que enfrentan para conseguir un trabajo remunerado, optan por la incorporación al sector informal. Esta realidad, queda retratada en el análisis de las condiciones de vida de las mujeres que se encuentran privadas de libertad. Solo el $15 \%$ de las mujeres recluidas trabajaban en comercio formal, en cambio, un 85 $\%$ se desempeñaba en actividades informales como ventas ambulantes, labores domésticas remuneradas y servicios (Fundación Arias para la Paz y el Progreso Humano, 2016).

De acuerdo a este trabajo, el $44 \%$ de las entrevistadas tenían ingresos mensuales menores a 100 mil colones. Por otro lado, no puede dejar de mencionarse que cerca de un $70 \%$ de las mujeres prisionalizadas, según datos del Ministerio de Justicia y Paz lo están por delitos patrimoniales o de tráfico de drogas, ilícitos que suelen vincularse a la pobreza (Feoli, 2019). Lo más grave es que la mayoría, en el caso de estupefacientes, fueron ventas al menudeo; es decir, no se caza a quienes integran los niveles más altos de una organización criminal sino a los últimos o a las últimas del eslabón delincuencial. En el mismo sentido, en la investigación realizada por la Fundación Arias para la Paz y el Progreso Humano en el 2016, un $71 \%$ de las mujeres entrevistadas expresaron haber tenido dificultades para encontrar trabajo antes de ingresar al centro, el $75 \%$ refirió vivir en situación de pobreza. Apenas un $32 \%$ era reincidente.

Un elemento de la mayor importancia que se debe tomar en cuenta al abordar los procesos penales contra las mujeres se relaciona con su mayor vulnerabilidad en relación con la violencia. Esto logra acreditarse en el "Diagnóstico realizado 
en el CAI Buen Pastor en 2015" (ahora llamado Vilma Curling Rivera). Casi la mitad de las entrevistadas señaló haber sido víctima de violencia de género: de violencia emocional un $35 \%$ y de violencia física el $32 \%$. Esto es, 6 de cada 10 mujeres presas fueron víctimas de violencia.

Finalmente, de acuerdo con la información aportada por las mujeres, el ingreso a prisión tuvo un impacto directo sobre la situación familiar: un $26 \%$ afrontó pobreza extrema, un 49 \% sufrió algún tipo de afectación emocional, un $13 \%$ cayó en exclusión escolar y un 17 \% manifestó que la reclusión de la mujer generó la separación de las personas que integraban el grupo familiar. Debe insistirse en el perfil criminológico de las reclusas: de las mujeres prisionalizadas solo un $11 \%$ se encuentra allí como consecuencia de haber cometido delitos contra la vida. Por su parte, alrededor de un $26 \%$ descuenta condenas por delitos contra la propiedad. Finalmente, un $54 \%$ de las mujeres presas están encerradas por delitos relacionados con la ley de psicotrópicos. Las demás categorías delictivas combinadas aportan el restante 9 \% (Dirección General de Adaptación Social, 2018). Dicho de otro modo, los patrones delictivos parecen desaconsejar la necesidad de que a las mujeres se les albergue, como ocurre hoy día, en módulos cuyo diseño apunta a reclusos de alta peligrosidad.

En definitiva, puede decirse que las mujeres que delinquen reflejan una estructura social desigual, lo cual queda evidenciado en las condiciones de vida previas a la comisión de un ilícito. Por lo tanto, es necesario que el sistema penal incorpore herramientas de género que procuren equilibrar situaciones de desventaja.

\section{La reforma esperada o la expresión del Nuevo Humanismo}

En noviembre de 2018, se aprobó la ley 9628 mediante la cual se reformaron los artículos 71 y 72 del Código Penal. Esta ley fue publicada por la Asamblea Legislativa (2019). Los ordinales modificados establecen lo siguiente:

\section{LA ASAMBLEA LEGISLATIVA DE LA REPÚBLICA DE COSTA RICA} DECRETA:

MODIFICACIÓN DE LOS ARTÍCULOS 71 Y 72 DE LA LEY N. ${ }^{\circ} 4573$, CÓDIGO PENAL, DE 4 DE MAYO DE 1970

ARTíCULO 1- Adición del inciso g) al artículo 71 de la Ley N. ${ }^{\circ} 4573$, Código Penal Se adiciona el inciso g) al artículo 71 de la Ley N. ${ }^{\circ} 4573$, Código Penal, de 4 de mayo de 1970. 
El texto es el siguiente: Modo de fijación Artículo 71- [...] g) Que la persona sentenciada sea una mujer que se encuentre en estado de vulnerabilidad, por pobreza, por tener bajo su responsabilidad el cuido y la manutención de familiares dependientes, por discapacidad o por ser víctima de violencia de género, cuando ese estado haya influido en la comisión del hecho punible.

ARTíCULO 2- Reforma del artículo 72 de la Ley N. ${ }^{\circ} 4573$, Código Penal Se reforma el artículo 72 de la Ley N. ${ }^{\circ} 4573$, Código Penal, de 4 de mayo de 1970.

El texto es el siguiente: Concurrencia de atenuantes y agravantes Artículo 72- Cuando concurran circunstancias agravantes y atenuantes en el mismo hecho punible, el juez las apreciará por su número e importancia, de acuerdo con el artículo anterior. Cuando concurra alguna de las circunstancias previstas en el inciso g) del artículo anterior y la mujer sentenciada no tenga antecedentes penales, el tribunal de juicio podrá disminuir la sanción, incluso por debajo del monto mínimo previsto en el tipo penal.

Como se veía, muchas de las mujeres que delinquen lo hacen por delitos de narcomenudeo (Palma, 2011) que se explican en la pobreza, pero esto también lleva a debatir sobre la irracionalidad que significa prisionalizar a personas que, por lo general, tienen un perfil criminológico de baja peligrosidad para las cuales el encierro implica un trastorno mayor no solo para la propia condenada, sino también para sus familias que es, en un buen número, un entorno a cargo de la mujer sentenciada (Coyle, 2009).

La reforma permite que en un juicio, en el momento de sancionar, los jueces puedan recortar la pena, esto es, rebajarla, en aquellos casos en los que la procesada no tenga antecedentes penales, es decir, que sea la primera vez que delinca, y logre probar que se encontraba en una condición de vulnerabilidad cuando cometió el delito. Para Feoli:

...con la reforma (...) los jueces cuando analicen un proceso criminal tendrán la posibilidad de rebajar la pena a aquellas mujeres, sin antecedentes, que en condición de vulnerabilidad, como pobreza, dependencia o violencia de género, cometieron un delito (...) Tanta irracionalidad se corregirá a partir de ahora, corresponderá a los jueces, mediante la prueba que aporten las partes, determinar cuándo una condición de vulnerabilidad influyó en 
la comisión del delito y qué tanto el grado de reproche debe adecuarse a las circunstancias. (2019)

En efecto, no se trata de una reducción automática de penas, deberá demostrarse que la condición de vulnerabilidad de las mujeres fue un elemento que las empujó a cometer el delito. Esta no es la primera reforma que se aprueba. En el año 2013 se modificó la Ley de Estupefacientes de Costa Rica, que castiga la venta y el tráfico de drogas. Sin embargo, este cambio legal fue bastante más modesto, pues solo se aplicó al delito de introducción de drogas en los centros penales. Se trata, en toda regla, de la aprobación de una acción afirmativa o de discriminación positiva. Conviene recordar que:

...la acción afirmativa es una norma legal, o una política pública que pretende lograr la igualdad de oportunidades para las mujeres. En breve consiste en escoger en una situación de paridad, a la persona que pertenezca a un sector discriminado, cualquiera que éste sea. Por tanto, requiere de un cambio de mentalidad de la sociedad en su conjunto. (Begné, 2012, p.16)

Hay dos elementos que merece la pena destacar de la reforma: por un lado, que lograra articularse la convicción sobre la necesidad de ver con otros ojos a las mujeres que delinquen e introducir fórmulas de discriminación positiva; por el otro, en un plano más ideológico y filosófico, la adopción de las ideas propias del Nuevo Humanismo (Barahona y Mata, 2015). Se reconoce el valor el ser humano y se superan las viejas categorías formales de igualdad que pueblan los textos legales. En su lugar, se atienden las diferencias que existen entre las personas, todas ellas estructurales, para impulsar cambios sociales anclados en valores como la justicia, la solidaridad y la dignidad.

\section{Desafíos pendientes en materia de mujeres en conflicto con la ley penal}

No hay duda de que en los últimos años ha habido una preocupación por visibilizar la realidad de las mujeres que entran en conflicto con la ley penal, conocer las razones que explican su actuar delictivo y procurar cambios que mejoren su atención y, sobre todo, que las aleje de la posibilidad de delinquir o de volver a hacerlo. Existen instrumentos internacionales, como las Regla Bangkok (Regla 45), que ordenan a los estados, dar un tratamiento diferenciado a los grupos más vulnerables. 
Ciertamente, aún quedan desafíos pendientes. Es probable que la reforma de los artículos 71 y 72 del Código Penal reduzca el número de mujeres privadas de libertad. Esto es, per se, una ganancia en términos de evitar la marginalidad y la exclusión de ciertos sectores. Sin embargo, es insuficiente.

Es necesario que se vertebren, a través de las distintas instituciones, iniciativas que tiendan la mano a las personas que continúan en riesgo. También que los programas que se han creado para atender a las mujeres que ya se encuentran dentro del sistema penitenciario (Reglamento del Sistema Penitenciario, 2018) sirvan no solo para reprimir, sino también para generar posibilidades reales de retornar a la vida en libertad en condiciones dignas. La reforma ayudará a que el Estado atenúe el castigo o, incluso, no ordene la prisionalización de una mujer que delinquió por encontrase en una situación de vulnerabilidad. Como sea, es solo una oportunidad para evitar el encierro, quizás momentáneamente.

Allí está el principal desafío, si el sistema penal cuenta hoy con una herramienta para distinguir la trágica realidad de muchas mujeres. No podemos esperar a que esa distinción sea simplemente temporal y que haya nuevas infracciones que contribuyan, ahora sí, a que se case con furia a quienes se quedaron sin alternativas. De ser así, de poco habría servido el cambio legal.

Hay muchas historias como las de María. Conjurar el riesgo de que se repitan pasa por fortalecer las políticas sociales de tal forma que se puedan cerrar las brechas que colocan a la región como la más desigual del planeta. Se debe cortar, definitivamente, con el peligro de que los grupos vulnerables acaben excluidos a través de la cárcel.

\section{Referencias}

Asamblea Legislativa. (2013). Ley 8204. San José, Costa Rica.

Asamblea Legislativa. (2018). Ley 9628. San José, Costa Rica.

Asamblea Legislativa de la República de Costa Rica. (2019). La Asamblea Legislativa de la República De Costa Rica decreta: modificación de los artículos 71 y 72 de la ley $n{ }^{\circ} 4573$, código penal. Recuperado de: http://derechoaldia.com/index.php/penal/ penal-leyes-usuales/1030-la-asamblea-legislativa-de-la-republica-de-costa-ricadecreta-modificacion-de-los-articulos-71-y-72-de-la-ley-n-4573-codigo-penal

Barahona, M. y Mata, E. (2015). Los Estudios Generales como pedagogía multidisciplinaria en vías a la interdisciplinar. Revista Nuevo Humanismo, 3 (1), 39-53. 
Begné, P. (2012). Acción afirmativa: una vía para reducir la desigualdad. Revista Ciencia Jurídica, 1 (1), 11-16.

Coyle, A. (2009). La prisión del futuro. En: Carranza, E. Cárcel y justicia penal en América Latina y el Caribe. San José, Costa Rica: ILANUD.

Davis, A. (2016). Democracia en abolición: prisiones racismo y violencia. Madrid, España: Editorial Trotta.

Dirección General de Adaptación Social. (2018). Información estadística sobre población penal. Recuperado de: www.mjp.go.cr

Facio, A y Fries, L. (2005). Feminismo, Género y Patriarcado. Revista sobre enseñanza del derecho, (6), 259-294.

Feoli, M. (2019). Algo de justicia para las mujeres. Recuperado de: https:// www.nacion.com/opinion/foros/algo-de-justicia-para-las-mujeres/ QOM32HXGYRGQREXOBKJMGMOZKM/story/

Fundación Arias para la Paz y el Progreso Humano. (2016). Proyecto “Mejoras al acceso a los derechos humanos de las mujeres jóvenes y adultas privadas de libertad por delitos relacionados con el tráfico de drogas y sus círculos sociales más cercanos. Recuperado de: http://arias.or.cr/presentamos-los-resultados-de-nuestro-proyecto-enfocado-en-mujeres-privadas-de-libertad-por-delitos-relacionados-con-el-trafico-de-drogas

Ministerio de Justicia y Paz. (2015). Diagnóstico de necesidades de mujeres privadas de libertad en el Centro de Atención Institucional (CAI) El Buen Pastor.

Oficina de las Naciones unidas contra la droga y el delito. (2014). Manual sobre estrategias para reducir el hacinamiento en prisiones. Recuperado de: https://www. unodc.org/documents/justice-and-prison-reform/HBonOvercrowding/UNODC HB_on_Overcrowding_ESP_web.pdf

Oficina de las Naciones unidas contra la droga y el delito. (2015). Reglas Mínimas de las Naciones Unidas para el Tratamiento de las Reclusas o Reglas Bangkok. Recuperado de: https://www.unodc.org/documents/justice-and-prison-reform/Bangkok_Rules_ESP_24032015.pdf

Palma Campos, C. (2011). Delito y sobrevivencia: las mujeres que ingresan a la cárcel El Buen Pastor en Costa Rica por tráfico de drogas. Anuario de Estudios Centroamericanos, (37), 245-270.

Pásara, L. (2013). Una reforma imposible: la justicia latinoamericana en el banquillo. Quito, Ecuador: Ediciones Legales EDLE S.A. 
Poder Judicial. (2018) ¿Cuáles son las condiciones de vulnerabilidad? Recuperado de: https://www.poder-judicial.go.cr/accesoalajusticia/index.php/ noticias/80-cuales-son-las-condiciones-de-vulnerabilidad-4

Reglamento del Sistema Penitenciario. (2018). Decreto Ejecutivo número 40849-JP. Costa Rica. 